\title{
Statistical size scaling of breakage strength of irregularly-shaped particles
}

\author{
Guian Qian ${ }^{\mathrm{a}}$, Wei-Sheng Lei ${ }^{\mathrm{b}, *}$, Zhishui Yu${ }^{\mathrm{c}, *}$, Filippo Berto ${ }^{\mathrm{d}}$ \\ ${ }^{a}$ State Key Laboratory of Nonlinear Mechanics (LNM), Institute of Mechanics, Chinese Academy of Sciences, Beijing 100190, China \\ ${ }^{\mathrm{b}}$ Applied Materials, Inc., 974 East Arques Avenue, Sunnyvale, CA 94085, USA \\ c School of Materials Engineering, Shanghai University of Engineering Science, 333 Long Teng Rd., Shanghai 201620, China \\ ${ }^{\mathrm{d}}$ Department of Mechanical and Industrial Engineering, Norwegian University of Science and Technology (NTNU), Richard Birkelands vei $2 b, 7491$ Trondheim, Norway
}

\section{A R T I C L E I N F O}

\section{Keywords:}

Irregularly-shaped particle

Breakage strength

Size effect

Spatial flaw distribution

Weakest- link model

Weibull statistics

\begin{abstract}
A B S T R A C T
The microdefects in a material responsible for fracture initiation in particle breakage may not always have a uniform spatial distribution. This work applies the power-law spatial flaw distribution based weakest link formulation of cumulative failure probability for statistical size scaling of breakage strength of irregularly-shaped particles. Four sets of published breakage strength data of different sized grains made of different materials including rock, basalt, sugar, and potash are adopted to validate the power-law spatial flaw distribution based weakest link model. Since the power-law spatial flaw distribution based weakest link model encompasses the uniform spatial flaw distribution based weakest link model as its subordinate member, while the Weibull statistical strength theory is a special case of the uniform spatial flaw distribution based weakest link model, this work extends the capability to investigate size scaling of particle breakage strength.
\end{abstract}

\section{Introduction}

Particle size reduction via breakage and fragmentation is an elementary physical event in particle comminution and powder granulation processes. The resistance to particle size reduction via breakage and fragmentation is usually measured by particle breakage strength. For brittle and quasi-brittle breakage of particles, the particle breakage strength features both random variation and particle size effect. The random variation of breakage strength is observed in a same compression experiment of particles with nominally the same geometrical shape and dimension. The size effect of particle strength refers to the inverse dependence of (average) particle strength on particle size. Both characteristics result from the random distribution of microdefects in particles with respect to their spatial location, orientation, size and shape. The effect of particle size lies in a change in the number of these microdefects in different sized particles. The random variation of strength calls for statistical assessment of the breakage strength of particles, especially irregularly-shaped particles. The statistical evaluation of particle strength falls into two methods, namely the empirical data fitting and the weakest link statistics, as summarized in Fig. 1.

- Empirical data fitting. So far, a variety of cumulative distribution functions (CDFs) has been adopted to describe the statistical behavior of breakage strength or energy of individual particles [1-7]. For example, recently, Cavalcanti and Tavares [7] used 12 CDFs for statistical analysis of fracture strength data of 5 industrial iron ore pellets in 5 size ranges: normal distribution, Gumbel distribution, logistic distribution, two- and three- parameter Weibull distributions, two- and three- parameter Gamma distributions, two- and three- parameter log-logistic distributions, log-normal distributions without truncation and with either lower or upper truncations. Some of the frequently used CDFs are listed below:

(1). Normal distribution: $P\left(\sigma_{b}\right)=\int_{-\infty}^{\sigma_{b}} \frac{d t}{\sqrt{2 \pi} \delta} \exp \left[-\left(\frac{t-\mu}{\sqrt{2} \delta}\right)^{2}\right]$

(2). Gumbel distribution: $P\left(\sigma_{b}\right)=\exp \left\{-\exp \left[-\left(\frac{\sigma_{b}-\sigma_{L}}{\sigma_{0}}\right)\right]\right\}$

(3). Logistic distribution: $P\left(\sigma_{b}\right)=\frac{1}{1+\exp \left[-\left(\frac{\sigma_{b}-\mu}{\sigma_{0}}\right)\right]}$

(4). $\log$ - normal distribution

$$
: P\left(\sigma_{b}\right)=\int_{0}^{\sigma_{b}} \frac{d t}{\sqrt{2 \pi} \delta t} \exp \left[-\left(\frac{\ln (t)-\mu}{\sqrt{2} \delta}\right)^{2}\right]
$$

\footnotetext{
* Corresponding authors.

E-mail addresses: Leiws2008@gmail.com (W.-S. Lei), yu_zhishui@163.com (Z. Yu).
} 


\begin{tabular}{|llll|}
\hline \multicolumn{2}{|l|}{ Nomenclature } & $\mathrm{d} V$ & $\begin{array}{l}\text { differential volume element } \\
\text { a reference volume }\end{array}$ \\
$\mathrm{g}(\mathrm{S})$ & probability density function of microscopic cleavage & $\sigma$ & $\begin{array}{l}\mathrm{V}_{0} \\
\text { local stress at any point inside } \mathrm{V} \\
\text { threshold of breakage strength }\end{array}$ \\
& strength $(\mathrm{S})$ & $\sigma_{\mathrm{L}}$ & nominal particle breakage strength \\
$\mathrm{m}$ & shape parameter & $\sigma_{b}$ & $\sigma_{1}, \sigma_{2}, \sigma_{3}$ first, second, third principal stress \\
$\mathrm{n}$ & total number of samples in each group & $\sigma_{0}$ & scale parameter \\
$N(V)$ & number of microcraks in a volume $V$ & $\sigma_{u}$ & scale parameter \\
$p\left(V_{0}\right)$ & fracture probability of $\mathrm{V}_{0}$ & $\sigma$ & mean stress value \\
$P\left(\sigma_{b}\right)$ & cumulative probability of failure at $\sigma_{b}$ & $\beta$ and $k$ constants \\
$\mathrm{PDF}$ & probability density function & $\Gamma(m)$ & Gamma function, \\
$\mathrm{S}$ & fracture strength of $\mathrm{V}_{0}$ & $\mu$ and $\delta$ mean value and standard deviation \\
$\mathrm{V}$ & specimen volume & & \\
\hline
\end{tabular}

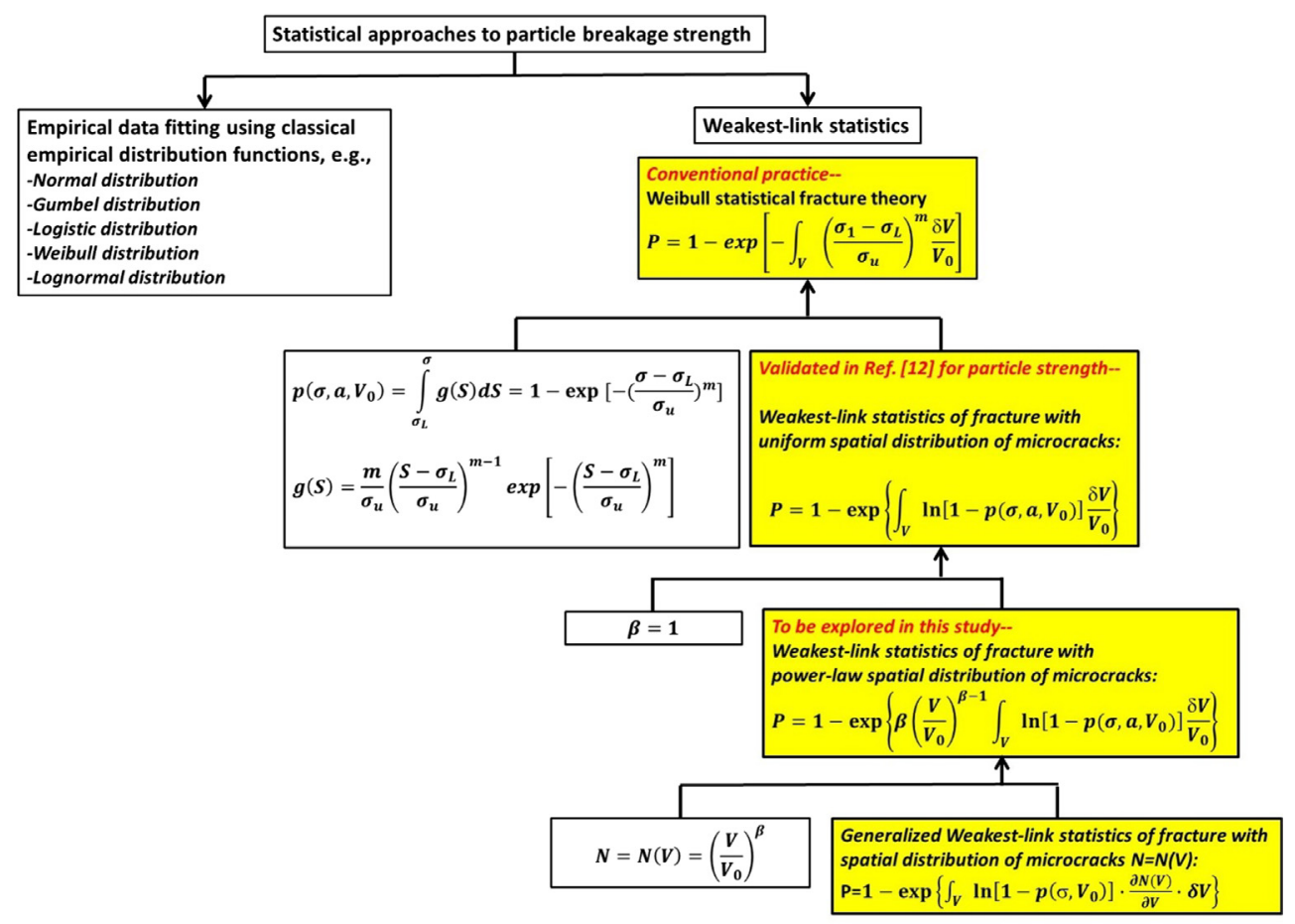

Fig. 1. Hierarchical relationships between Weibull statistical fracture theory and weakest-link statistics under different assumptions of spatial distribution of microdefects in a material.

$$
\text { (5). } \log -\text { logistic distribution: } P\left(\sigma_{b}\right)=\frac{1}{1+\exp \left\{-\left[\frac{\ln \left(\sigma_{b}\right)-\mu}{\sigma_{0}}\right]\right\}}
$$

(6). Three - parameter Weibull distribution

$$
: P\left(\sigma_{b}\right)=1-\exp \left[-\left(\frac{\sigma_{b}-\sigma_{L}}{\sigma_{0}}\right)^{m}\right]
$$

(7). Three - parameter Gamma distribution

$$
: P\left(\sigma_{b}\right)=\int_{0}^{\sigma_{b}} \frac{\left(t-\sigma_{L}\right)^{m-1} \exp \left[-\left(\frac{t-\sigma_{L}}{\sigma_{0}}\right)\right] d t}{\Gamma(m) \sigma_{0}^{m}}
$$

where $P\left(\sigma_{b}\right)$ is the cumulative probability of failure at the nominal particle breakage strength $\sigma_{b}, \sigma_{L}$ is the threshold of breakage strength, $\sigma_{0}$ is the scale parameter, $m$ is the shape parameter, $\Gamma(m)$ is the Gamma function, $\mu$ and $\delta$ are the mean value and standard deviation of the absolute value or the logarithmic value of breakage strength $\sigma_{b}$, respectively.

Usually, the distribution parameters ( $\mathrm{m}, \sigma_{0}, \sigma_{L}, \mu$ and $\delta$ ) are estimated by the least-squares and the maximum likelihood methods, while the goodness-of-fit of probability distributions to data is analyzed by some tests such as the Anderson-Darling (A-D) test and the
Kolmogorov- Smirnov (K-S) test.

The empirical data fitting approach works well for strength data collected from a group of nominally same sized particles. All the CDFs in Eqs. (1)-(7) do not contain specimen size as an explicit variable. As particle size changes, empirical data fitting will be applied for each group of particles with same size. The size effect is reflected on the variation of the fitted distribution parameters with particle size. The empirical fitting approach is of practical significance for statistical description of a given sized particle. However, since it is not based on physical understanding of particle breakage processes, the obtained empirical fitting formulae do not have prediction power for other different sized particles.

- Weakest link statistics. The weakest link statistics assumes that the strength of a bulk solid is determined by its weakest volume element. Initially, Weibull applied the weakest link assumption and obtained the following expression [8]:

$P=1-\exp \left[-\int_{V}\left(\frac{\sigma-\sigma_{L}}{\sigma_{u}}\right)^{m} \frac{d V}{V_{0}}\right]$

where $\sigma$ is the local stress at any point inside a specimen of volume $\mathrm{V}$, $\mathrm{V}_{0}$ is a reference volume, $\mathrm{dV}$ is a differential volume element, $\sigma_{u}$ is a 
scale parameter. Under the uniform stress condition, $\sigma=\sigma_{b}$, Eq. (8) reduces to

$P=1-\exp \left[-\frac{V}{V_{0}}\left(\frac{\sigma_{b}-\sigma_{L}}{\sigma_{u}}\right)^{m}\right]=1-\exp \left[-\left(\frac{\sigma_{b}-\sigma_{L}}{\sigma_{0}}\right)^{m}\right]$

with $\sigma_{0}=\sigma_{u}\left(V_{0} / V\right)^{1 / m}$.

According to Eq. (8), the weakest link assumption permits to represent both the random variation of nominal breakage strength $\left(\sigma_{b}\right)$ and the size effect in the form of specimen volume $(V)$ in the cumulative failure probability formulation.

Note that Weibull statistics of strength obeys the weakest-link assumption. In other words, Weibull statistics is only a subordinate member of the weakest-link statistics, as shown in Fig. 1 [9-11]. Therefore, it makes sense to directly resort to the weakest-link statistics for particle strength evaluation. According to $[9,10]$, the explicit weakest-link formulation for the cumulative probability of brittle fracture under the assumption of uniform spatial distribution of microdefects is as follows:

$P(V)=1-\exp \left[\int_{\mathrm{v}} \ln \left[\left(1-p\left(V_{0}\right)\right] \frac{d V}{V_{0}}\right]\right.$

where $p\left(V_{0}\right)$ is the fracture probability of an elemental volume $\mathrm{V}_{0}$ induced by an embedded microcrack in a multiaxial stress state described by the principal stresses $\left(\sigma_{1}, \sigma_{2}, \sigma_{3}\right)\left(\sigma_{1} \geq \sigma_{2} \geq \sigma_{3}\right)$.

When the probability density function (PDF) with respect to the fracture strength (S) of elemental volume $\mathrm{V}_{0}$, denoted as $\mathrm{g}(\mathrm{S})$, takes the well-known three-parameter Weibull PDF as

$g(S)=m\left[\left(S-\sigma_{L}\right)^{m-1} / \sigma_{u}^{m}\right] \cdot \exp \left[-\left(S-\sigma_{L}\right)^{m} / \sigma_{u}^{m}\right] \quad\left(m>0, \sigma_{L} \leq S<\infty\right)$

under the maximum principal tensile stress criterion $\sigma_{1}=\sigma \geq S$, there is

$p\left(\sigma_{1}, V_{0}\right)=\int_{\sigma_{L}}^{\sigma} g(S) d S=1-\exp \left[-\left(\frac{\sigma-\sigma_{L}}{\sigma_{u}}\right)^{m}\right]$

Substitution of Eq. (12) in Eq. (10) leads to Eq. (8). This proves that Weibull statistics is a subordinate member of weakest-link statistics.

Basically, depending on the specific material, $p\left(V_{0}\right)$, as the fracture probability of an elemental volume $\mathrm{V}_{0}$, can take any other distribution function, such as the CDFs in Eqs. (1)-(7). But in reality, the exact strength distribution function $\mathrm{g}(\mathrm{S})$ and the pertinent microscopic fracture criterion are unknown. Therefore, it is not sure which CDF best represents $p\left(V_{0}\right)$ for a specific material. As an alternative solution, Eq. (10) is rewritten as below due to the first mean value theorem for integrals,

$\frac{1}{V} \cdot \ln \left[\frac{1}{(1-P)}\right]=\frac{1}{V_{0}} \cdot \ln \left[\frac{1}{1-p\left(\sigma, V_{0}\right)}\right]=h\left(\sigma_{b}\right)$

where $\sigma$ is a mean stress value with $\sigma_{L} \leq \sigma \leq \sigma_{b}$. Therefore, in Eq. (13), the compound parameter $\frac{1}{V} \cdot \ln \left[\frac{1}{(1-P)}\right]$ is also conceptually expressed as function of particle strength $h\left(\sigma_{b}\right)$. By evaluating the correlation between the compound parameter $\frac{1}{V} \cdot \ln \left[\frac{1}{(1-P)}\right]$ and particle strength $\sigma_{\mathrm{b}}$, the twofold character of brittle failure strength $\sigma_{\mathrm{b}}$ can be evaluated together.

The expected "master curve" for the $\frac{1}{V} \cdot \ln \left[\frac{1}{(1-P)}\right] \sim \sigma_{b}$ correlation in Eq. (13) was validated by breakage strength data of granular particles made of glass, soil, salt, and copper ores in [12].

However, it was found that for some quasi-brittle materials, Eq. (13) does not work for their size scaling of strength. In principle, the basic formulation for the weakest-link statistics of brittle fracture depends primarily on the spatial distribution of microdefects in a material among others. The real critical prerequisite for the spatial distribution is the mutual independence of microdefects, which justifies the applicability of the Griffith law and weakest-link assumption at individual microdefect and differential volume element scales. Therefore, it is unnecessary to confine our assumption to the uniform spatial distribution of flaws. So long as the principle of mutual independence of microdefects is observed, other spatial distributions of flaws are allowed. In view of these two aspects, the generalized weakest-link statistics was developed in [11], which encompasses the preceding uniform spatial microdefect distribution based weakest link formulation as its subordinate member, as illustrated in Fig. 1. The generalized weakest link statistics was validated for size scaling of strength of wood, concrete, coal, gamma titanium aluminum alloy, nuclear-grade graphite, and aluminum foam [11]. This work will evaluate the applicability of generalized weakest link statistics for size scaling of particle breakage strength. This will start with a brief description of the generalized weakest-link statistics. Then specific examples will be presented for size scaling of particle breakage strength according to the generalized weakest-link statistics.

\section{A description of the generalized weakest-link statistics for brittle fracture}

The reader is referred to [11] for a detailed derivation of the generalized weakest-link formulation for the cumulative probability of brittle fracture. Assume that all the microcracks in a material are noninteractive and $N(V)$ is the number of microcraks in a volume $V$. Consider a differential volume element $\delta V$ subjected to a homogeneous stress state $\left(\sigma_{1}, \sigma_{2}, \sigma_{3}\right)$, where $\sigma_{1}, \sigma_{2}$, and $\sigma_{3}$ are the three principal stresses and $\sigma_{1} \geq \sigma_{2} \geq \sigma_{3}$. The number of microcracks inside $\delta V$ is $\delta N=(\partial N / \partial V) \cdot \delta V$.The assumption of mutual independence of all the microcracks permits to apply the weakest-link postulate to an individual differential volume element scale. Under certain microscopic fracture criterion such as the maximum principal stress criterion, the failure probability of this differential volume element, $\delta P(\delta V)$, follows:

$\delta P(\delta V)=1-\left[1-p\left(\sigma, V_{0}\right)\right]^{\delta N}$

Next, for a bulk solid subjected to heterogeneous multiaxial stresses designated by $\Sigma\left(\sigma_{1}, \sigma_{2}, \sigma_{3}\right)$, its total volume $(V)$ is divided into many small volume elements $\delta V_{i}(i=1,2, \ldots, \mathrm{n})$, each of which is in a quasihomogeneous stress state. Then the cumulative failure probability of the solid, $P$, is

$P=1-\prod_{i=1}^{n}\left[1-\delta P_{i}\left(\delta V_{i}\right)\right]=1-\prod_{i=1}^{n}\left[1-p\left(\sigma, V_{0}\right)\right]^{\delta N_{i}}$

Eq. (15) is further written as

$$
\begin{aligned}
& P=1-\exp \left\{\sum_{i=1}^{n} \delta N_{i} \cdot \ln \left[1-p\left(\sigma, V_{0}\right)\right]\right\} \\
& \stackrel{n \rightarrow \infty}{\Rightarrow} 1-\exp \left\{\int_{V} \ln \left[1-p\left(\sigma, V_{0}\right)\right] \cdot \frac{\partial N(V)}{\partial V} \cdot \delta V\right)
\end{aligned}
$$

Eq. (16) is the explicit expression of the cumulative failure probability $P$ under the weakest-link postulate due to a population of mutually independent microcracks. In principle, so long as the mutual independence of microcracks is satisfied, Eq. (16) can adopt any specific distribution function of microcracks $N(V)$. The following power law is a relatively simple function to represent the spatial distribution of microdefects:

$N=N(V)=k V^{\beta}=\left(\frac{V}{V_{0}}\right)^{\beta}, \quad k=\frac{1}{V_{0}^{\beta}}, \beta>0$

where $\beta$ and $k$ are constants with $\beta, k>0 . k$ has the unit of $V^{-\beta}$ for dimensional consistency. Accordingly, the number of microcracks inside $\delta V$ will be,

$\delta N=\frac{\partial N(V)}{\partial V} \cdot \delta V=k \beta V^{\beta-1} \delta V=\beta\left(\frac{V}{V_{0}}\right)^{\beta-1}\left(\frac{\delta V}{V_{0}}\right)$

Substitution of Eq. (18) in Eq. (16) gives 

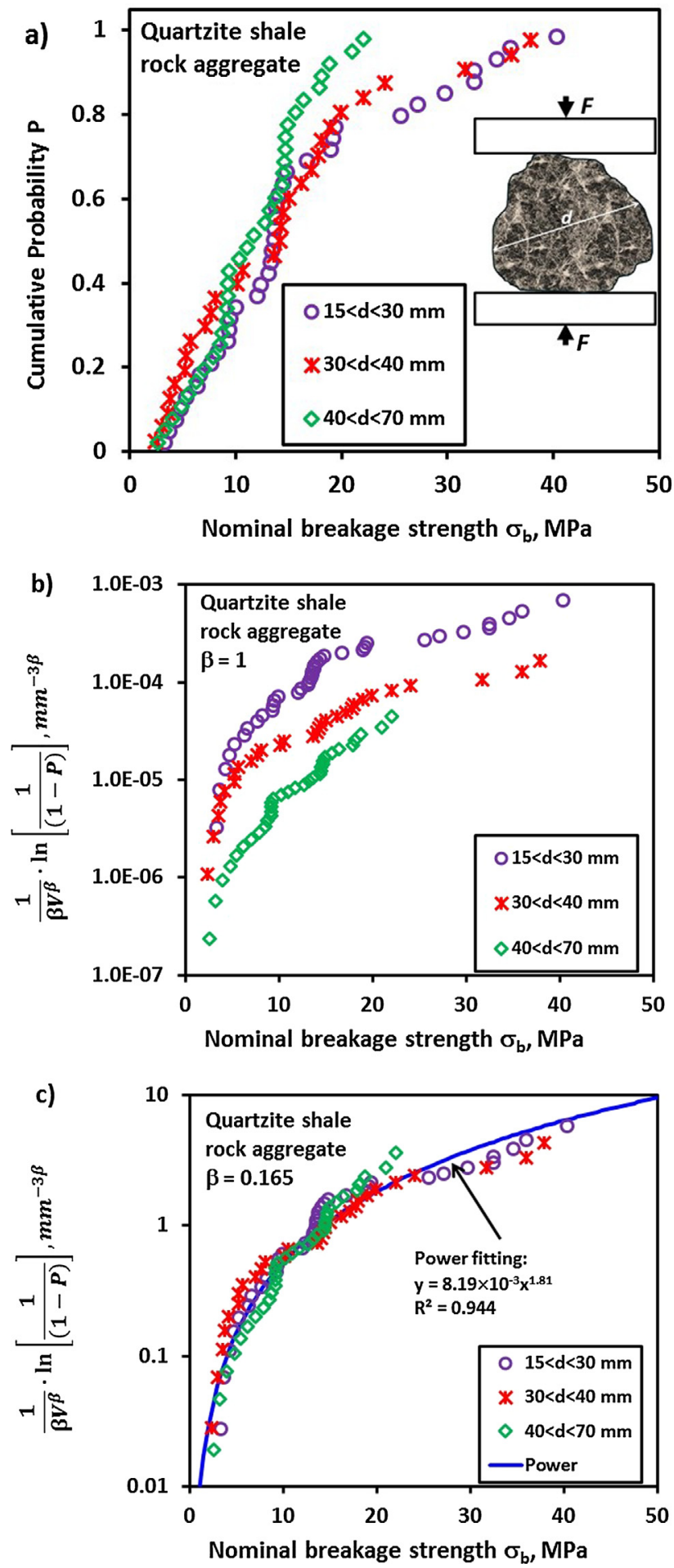

Fig.2. Breakage strength $\left(\sigma_{b}\right)$ of breakage strength of quartzite shale rock aggregates of 3 different size ranges: (a).raw data [3]; (b). correlation between $\frac{1}{V} \cdot \ln \left[\frac{1}{(1-P)}\right]$ and $\sigma_{b} ;$ (c). correlation between $\frac{1}{\beta V^{\beta}} \cdot \ln \left[\frac{1}{(1-P)}\right]$ and $\sigma_{b}$.

$P=1-\exp \left\{\beta\left(\frac{V}{V_{0}}\right)^{\beta-1} \int_{V} \ln \left[1-p\left(\sigma, V_{0}\right)\right] \cdot \frac{\delta V}{V_{0}}\right\}$

Eq. (19) is the expression for the power-law spatial microdefect distribution based weakest-link statistics.

Regardless of the complexity associated with the specific formulation of $p\left(\sigma, V_{0}\right)$, which depends on the specific PDF for the strength or size distribution of microdefects in a particle and the exact microscopic fracture criterion in addition to the stress state, our major interest here is the statistical characterization of size effect. So, similar to the case of uniform spatial distribution, according to the first mean value theorem for integrals, Eq. (19) is rewritten as

$\frac{1}{\beta V^{\beta}} \cdot \ln \left[\frac{1}{(1-P)}\right]=\frac{1}{V_{0}^{\beta}} \cdot \ln \left[\frac{1}{1-p\left(\xi, V_{0}\right)}\right]=\frac{h\left(\sigma_{b}\right)}{V_{0}^{\beta}}$

Accordingly, both the nominal strength variation and the size effect are now synchronized by the correlation between the compound parameter $\frac{1}{\beta V^{\beta}} \cdot \ln \left[\frac{1}{(1-P)}\right]$ and nominal strength $\sigma_{b}$. It suggests that for geometrically similar specimens of difference sizes under same loading conditions, there exists a master curve to correlate the compound parameter $\frac{1}{\beta V^{\beta}} \cdot \ln \left[\frac{1}{(1-P)}\right]$ and nominal strength $\sigma_{b}$.

When the PDF with respect to the fracture strength (S) of elemental volume $\mathrm{V}_{0}, g(S)$,takes the well-known Weibull PDF in Eq. (11), the corresponding formulation of $p\left(\sigma, V_{0}\right)$ under the maximum principal tensile stress criterion $\sigma_{1}=\sigma \geq S$ is given in Eq. (12). Substitution of Eq. (12) in Eq. (19) leads to

$P=1-\exp \left\{-\beta\left(\frac{V}{V_{0}}\right)^{\beta-1} \int_{V}\left(\frac{\sigma-\sigma_{L}}{\sigma_{u}}\right)^{m} \cdot \frac{\delta V}{V_{0}}\right\}$

When $\beta=1$, Eqs. (19) and (20) reduce to Eqs. (10) and (13) for uniform spatial distribution of microcracks, respectively, while Eq. (21) reduces to Weibull statistical fracture theory in Eq. (8). In other words, the uniform spatial microdefect distribution based weakest link formulation is a subordinate member of power-law spatial microdefect distribution based weakest link statistics, while it has Weibull statistical fracture theory as its subset.

It is noted that the model is limited to brittle fracture and does not include ductile fracture. Many advanced technological materials, such as metallic alloys, are usually ductile under normal conditions, but under extreme circumstances, including low temperature and dynamic loading conditions, can exhibit macroscopically brittle fracture behavior (with localized/microscopic plastic deformation). Ferritic steels commonly used to manufacture nuclear reactor pressure vessels and other engineering structures, can fail by cleavage fracture as a random event. In this case, statistical models have also been developed. For these technological materials, thanks to the significant progress in metallurgical industry, defects initiating brittle fracture, such as carbides and other nonmetallic inclusions in ferritic steels and cast irons, can be controlled to distribute uniformly in materials. Our studies have validated the uniform defect distribution based statistical models for cleavage fracture of ferritic steels. We believe and have also found that brittle and quasi-brittle materials, particularly those naturally grown materials, are more likely to have nonuniform spatial distribution of defects. Specifically, in Lei [11], it was found that wood and coal as natural materials, and gamma titanium aluminum alloy, aluminum foam and nuclear grade graphite as technological materials, can be better described with this nonuniform defection distribution based statistical model. In Lei [13], it was reported that the statistical distribution of defects in different ceramic materials can fit either $\beta=1$, $\beta>1$ or $0<\beta<1$.

In the next section, Eq. (20) will be adopted to establish the correlation between the compound parameter $\frac{1}{\beta V^{\beta}} \cdot \ln \left[\frac{1}{(1-P)}\right]$ and nominal breakage strength $\sigma_{b}$ of different sized particles made of different materials.

\section{Case studies on size dependence of particle breakage strength in compression}

In this section, we'll evaluate the particle breakage strength of several different materials.

The first example is about the strength of rock aggregates. Ovalle et al. [3] studied size effect on the strength of quartzite shale rock 
aggregates. The nominal particle breakage strength $\sigma_{b}$ was calculated as follows:

$\sigma_{b}=\frac{F}{d^{2}}$

where $\mathrm{d}$ is the mean value of the particle cross section diameter prior to compression and $\mathrm{F}$ is the diametrical compression force at fracture with the schematic illustration of experimental setup embedded in Fig. 2(a). The rank probability is adopted to measure the cumulative failure probability $\mathrm{P}=P\left(\sigma_{b, i}\right)$ at the $i$-th nominal breakage strength $\sigma_{b, i}$ by rearranging all the strength values in each group of nominally same sized particles in an ascending order,

$P\left(\sigma_{b, i}\right)=\frac{i-0.3}{n+0.4} \quad(1 \leq i \leq n)$

where $\mathrm{n}$ is the total number of samples in each group.

\subsection{Quartzite shale rock aggregates}

Fig. 2(a) shows the raw data of breakage strength of quartzite shale rock aggregates of 3 different size ranges namely, $15-30 \mathrm{~mm}$, $30-40 \mathrm{~mm}$, and $40-70 \mathrm{~mm}$ [3]. Fig. 2(b) shows the strength data rearranged according to Eq. (13), or Eq. (20) with $\beta=1$, for the uniform spatial flaw distribution based weakest link statistics. The three sets of strength data do not fall onto a single "master curve" for the $\frac{1}{V} \cdot \ln \left[\frac{1}{(1-P)}\right] \sim \sigma_{b}$ correlation, indicating the invalidity of the assumption of a uniform spatial distribution of flaws. Fig. 2(c) shows the strength data rearranged according to Eq. (20) with $\beta=0.165$. Now all the data fall onto a single master curve. Despite that there is a relatively large size interval of quartzite shale rock aggregates in each group, particularly the group of $40-70 \mathrm{~mm}$, the "master curve" behavior is very obvious. The correlation between the compound parameter $\frac{1}{\beta V^{\beta}} \cdot \ln \left[\frac{1}{(1-P)}\right]$ and nominal breakage strength $\sigma_{b}$ fits well to a power law function $y=8.19 \times 10^{-3} x^{1.81}\left(R^{2}=0.944\right)$. In equivalence, it can be expressed as

$P=1-\exp \left[-0.165\left(\frac{V}{V_{0}}\right)^{0.165}\left(\frac{\sigma_{b}}{14.2 M P a}\right)^{1.81}\right]\left(V_{0}=1 \quad \mathrm{~mm}^{3}\right)$

This supports that the spatial distribution of microdefects in the rock aggregates is non-uniform and can be approximately described by a power law function $N(V)=\left(V / V_{0}\right)^{0.165}$.

\subsection{Basalt grains}

Rozenblat et al. [2] studied the strength distributions of different sized particles made of eight (8) different materials namely, salt, potash, graphene nanoplatelets (GNP), glass spheres, sodium dodecyl sulfate (Sds), sugar, basalt, and marble, with limited strength data reported for Sds and marble. Except that glass spheres are regularly shaped, all other seven (7) types of particles are irregularly shaped. In their work [2], several tens of experiments were conducted for each particle size range for each material. However, only ten data points for each size range as the "diluted" experimental data but with actual corresponding rank probabilities were provided in order for clear graphical presentation. In the earlier work [12], the breakage strength data of different sized glass spheres, salt grains and GNP particles were analyzed to validate the expected "master curve" for the $\frac{1}{V} \cdot \ln \left[\frac{1}{(1-P)}\right] \sim \sigma_{b}$ correlation according to Eq. (13) for the uniform spatial flaw distribution based weakest link statistics. This work will analyze the breakage strength data of potash, sugar and basalt based on Eq. (20).

The nominal breakage strength $\sigma_{b}$ of a particle in diametrical compression is calculated as follows in [2]:

$\sigma_{b}=\frac{4 F}{\pi d^{2}}$
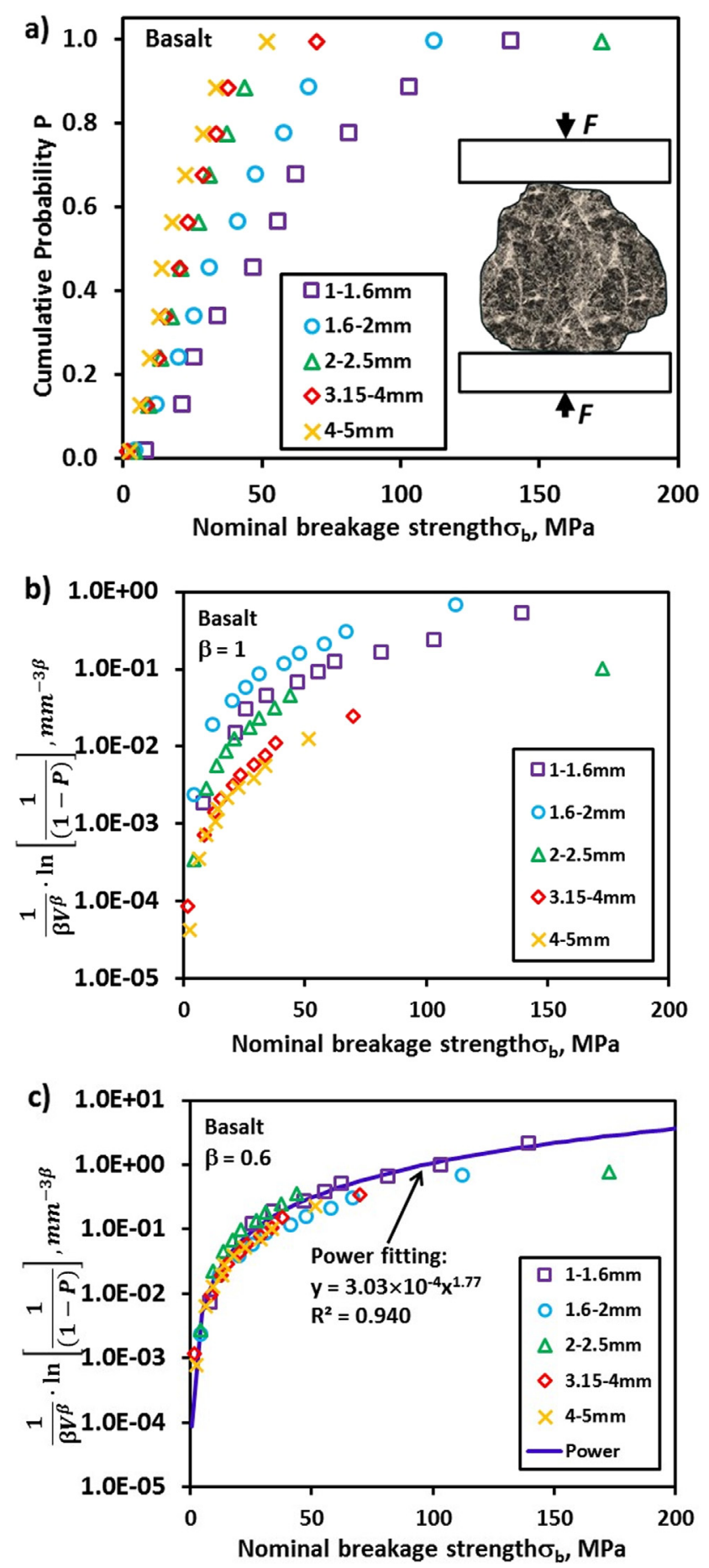

Fig.3. Breakage strength $\left(\sigma_{b}\right)$ of breakage strength of basalt grains: (a) raw data [2]; (b) correlation between $\frac{1}{V} \cdot \ln \left[\frac{1}{(1-P)}\right]$ and $\sigma_{b}$; (c) correlation between $\frac{1}{\beta V^{\beta}} \cdot \ln \left[\frac{1}{(1-P)}\right]$ and $\sigma_{b}$.

Fig. 3(a) shows the raw data of breakage strength of basalt grains of 5 different size ranges namely, $1-1.6 \mathrm{~mm}, 1.6-2 \mathrm{~mm}, 2-2.5 \mathrm{~mm}$, $3.15-4 \mathrm{~mm}$, and $4-5 \mathrm{~mm}$ [2]. First, the strength data are rearranged according to Eq. (13), or Eq. (20) with $\beta=1$, for the uniform spatial flaw distribution based weakest link statistics, as shown in Fig. 3(b). Obviously, a "master curve" for the $\frac{1}{V} \cdot \ln \left[\frac{1}{(1-P)}\right] \sim \sigma_{b}$ correlation does not exist. This suggests that the assumption of a uniform spatial distribution of flaws is inappropriate for basalt. Now the strength data are rearranged according to Eq. (20) with $\beta=0.6$, as shown in Fig. 3(c). 
The correlation between the compound parameter $\frac{1}{\beta V^{\beta}} \cdot \ln \left[\frac{1}{(1-P)}\right]$ and nominal breakage strength $\sigma_{b}$ fits well to a power law function $y=3.03 \times 10^{-4} x^{1.77}\left(R^{2}=0.940\right)$. In equivalence, it can be expressed as

$P=1-\exp \left[-0.6\left(\frac{V}{V_{0}}\right)^{0.6}\left(\frac{\sigma_{b}}{97.2 \mathrm{MPa}}\right)^{1.77}\right] \quad\left(V_{0}=1 \mathrm{~mm}^{3}\right)$

This supports that the spatial distribution of microdefects in basalt grains is non-uniform and can be approximately described by a power law function $N(V)=\left(V / V_{0}\right)^{0.6}$.

\subsection{Sugar grains}

Fig. 4(a) shows the raw data of breakage strength of sugar grains of 4 different size ranges namely, $1.6-2 \mathrm{~mm}, 2.5-3.15 \mathrm{~mm}, 3.15-4 \mathrm{~mm}$, and $4-5 \mathrm{~mm}$ [2]. Fig. 4(b) and (c) show the strength data rearranged according to Eq. (20) with $\beta=1$ for the uniform spatial flaw distribution based weakest link statistics and $\beta=0.4$ for the power law spatial flaw distribution based weakest link statistics. Again, a "master curve" for the $\frac{1}{V} \cdot \ln \left[\frac{1}{(1-P)}\right] \sim \sigma_{b}$ correlation does not exist, suggesting the inappropriate assumption of a uniform spatial distribution of flaws for sugar. Instead, the correlation between the compound parameter $\frac{1}{\beta V^{\beta}} \cdot \ln \left[\frac{1}{(1-P)}\right]$ and nominal breakage strength $\sigma_{b}$ fits well to a power law function $y=0.0203 x^{1.8881}\left(R^{2}=0.9344\right)$. In equivalence, it can be expressed as

$P=1-\exp \left[-0.4\left(\frac{V}{V_{0}}\right)^{0.4}\left(\frac{\sigma_{b}}{2.33 \mathrm{MPa}}\right)^{1.89}\right] \quad\left(V_{0}=1 \mathrm{~mm}^{3}\right)$

This supports that the spatial distribution of microdefects in sugar grains is non-uniform and can be approximately described by a power law function $N(V)=\left(V / V_{0}\right)^{0.4}$.

\subsection{Potash grains}

Fig. 5(a) shows the raw data of breakage strength of potash grains of 4 different size ranges namely, $1.4-2 \mathrm{~mm}, 2-2.36 \mathrm{~mm}, 2.36-3.35 \mathrm{~mm}$, and 3.35-4 mm [2]. Fig. 5(b) and (c) show the strength data rearranged according to Eq. (20) with $\beta=1$ and $\beta=0.53$, respectively. The uniform spatial distribution of flaws is an inappropriate assumption for potash. Instead, the correlation between the compound parameter $\frac{1}{\beta V^{\beta}} \cdot \ln \left[\frac{1}{(1-P)}\right]$ and nominal breakage strength $\sigma_{b}$ fits well to a power law function $y=0.0012 x^{3.152}\left(R^{2}=0.941\right)$. In equivalence, it can be expressed as

$P=1-\exp \left[-0.53\left(\frac{V}{V_{0}}\right)^{0.53}\left(\frac{\sigma_{b}}{8.45 \mathrm{MPa}}\right)^{3.15}\right] \quad\left(V_{0}=1 \mathrm{~mm}^{3}\right)$

This supports that the spatial distribution of microdefects in potash grains is non-uniform and can be approximately described by a power law function $N(V)=\left(V / V_{0}\right)^{0.53}$.

\section{Discussions}

The effect of particle size on particle breakage strength is of interest to many industrial processes. For regularly shaped spherical particles, analytical solutions to the elastic stress field inside a spherical body are available, which greatly help to understand their mechanistic breakage processes. However, for various irregularly shaped particles commonly encountered in different industries, analytical solutions to the elastic stress field inside them are unavailable. Empirical data accumulation and analysis becomes the major avenue to characterize their breakage strength. In this regard, the Discrete Element Method (DEM) enables to simulate the evolution process of defects in a granular object with strictly predefined geometry in terms of shape and size in a deterministic way. When the size and shape of a particle changes, a dedicated round of DEM simulation is needed. Therefore, statistical approaches are very attractive to characterize the size effect on breakage strength of irregularly shaped particles with various complexity of sphericity and roundness [6]. While the continued adoption of the classical empirical statistical distribution functions (e.g., Eqs. (1)-(7)) is expected for the empirical data fitting of breakage strength at each size range as a conventional practice, the physically justified weakest-link statistical approach has demonstrated its superior advantages in the aspect of size scaling. The $\beta$ parameter describes the degree of deviation of the spatial distribution of defects from the idealized uniform spatial distribution
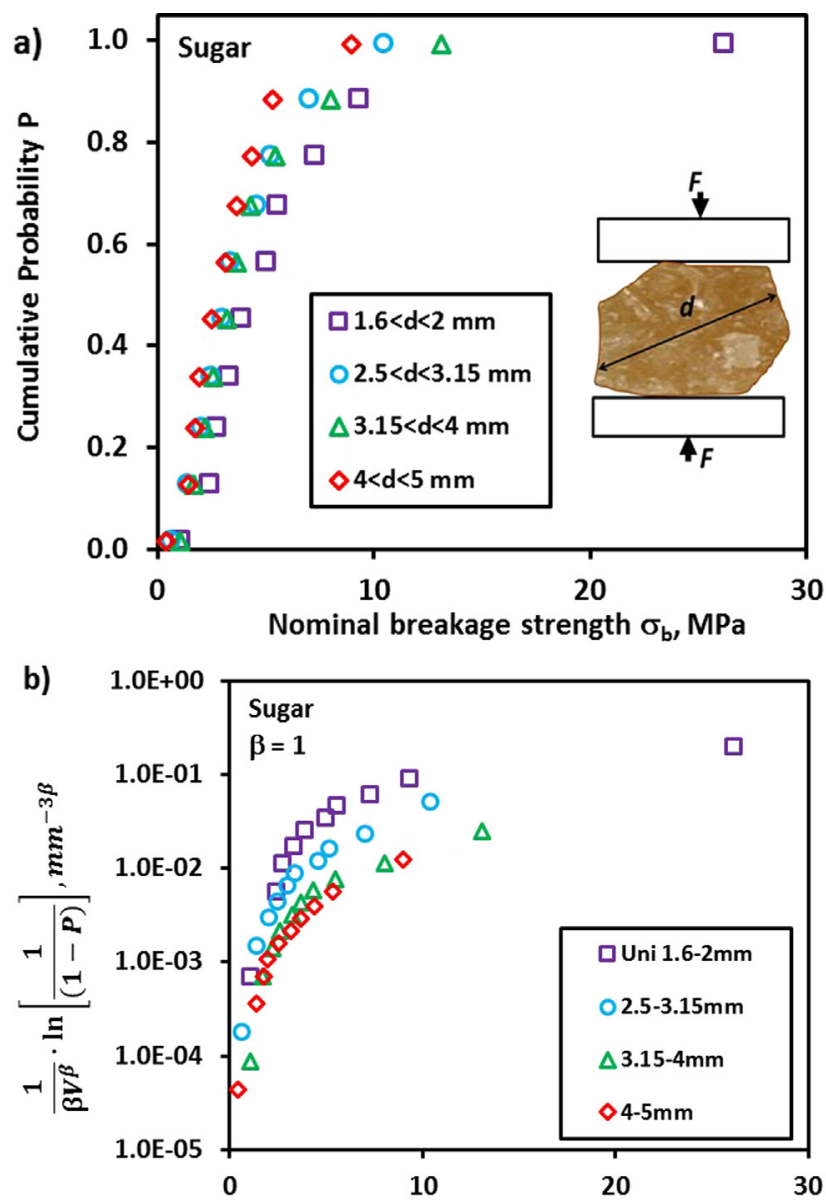

Nominal breakage strength $\sigma_{\mathrm{b}}, \mathrm{MPa}$

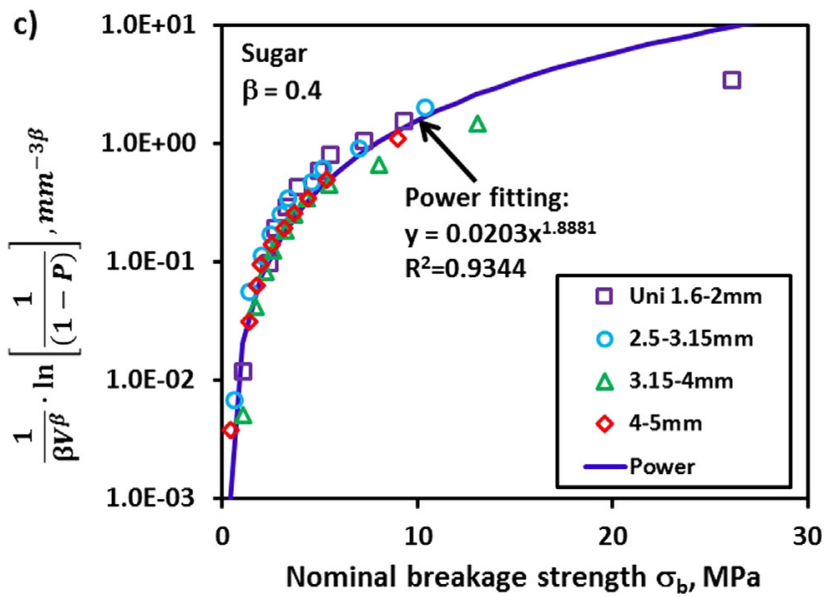

Fig.4. Breakage strength $\left(\sigma_{b}\right)$ of breakage strength of sugar grains: (a) raw data [2]; (b) correlation between $\frac{1}{V} \cdot \ln \left[\frac{1}{(1-P)}\right]$ and $\sigma_{b}$; (c) correlation between $\frac{1}{\beta V^{\beta}} \cdot \ln \left[\frac{1}{(1-P)}\right]$ and $\sigma_{b}$. 

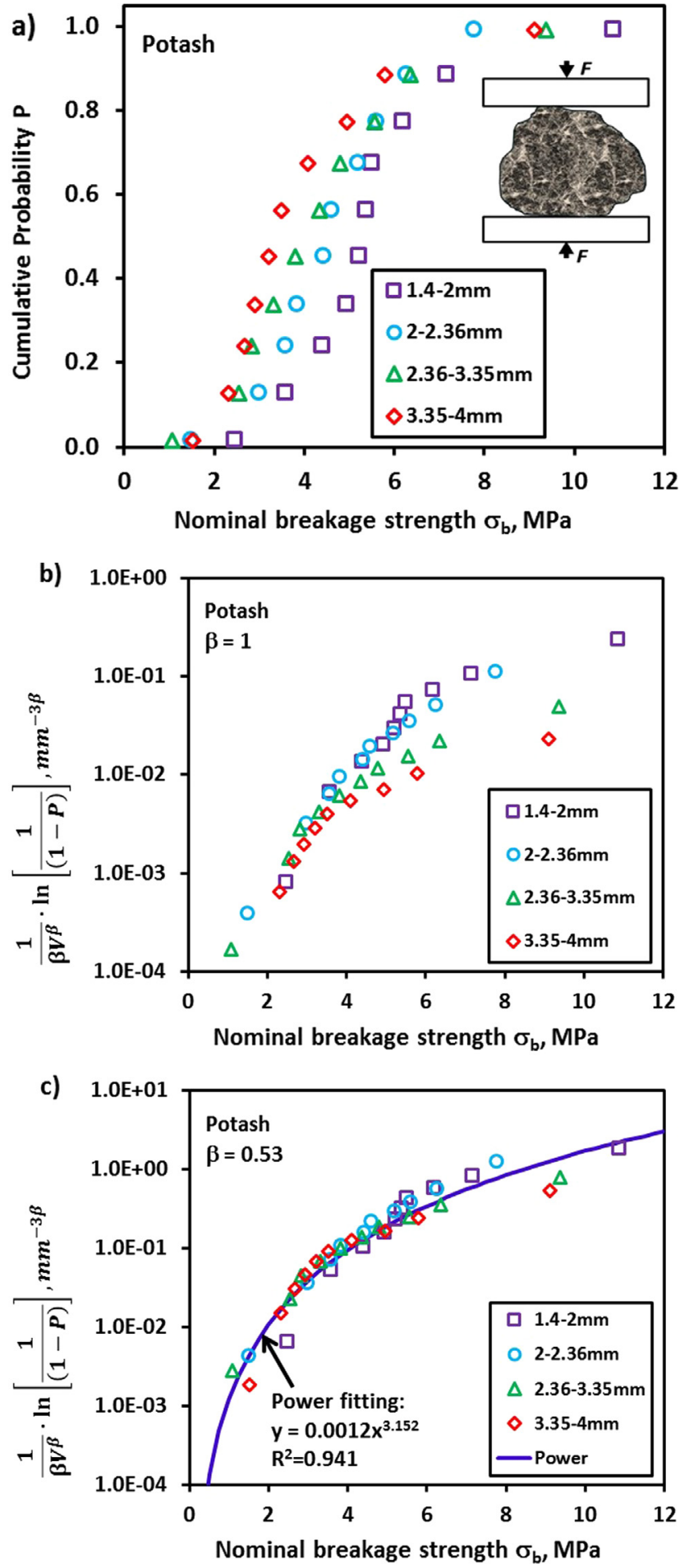

Fig.5. Breakage strength $\left(\sigma_{b}\right)$ of breakage strength of potash grains: (a) raw data [2]; (b) correlation between $\frac{1}{V} \cdot \ln \left[\frac{1}{(1-P)}\right]$ and $\sigma_{b}$; (c) correlation between $\frac{1}{\beta V \beta} \cdot \ln \left[\frac{1}{(1-P)}\right]$ and $\sigma_{b}$.

condition. If $\beta=1$, it is a perfect uniform distribution; if $0<\beta<1$, defects are less dense than uniform distribution and are nonuniform; if $\beta>1$, defects are more dense than uniform distribution and are nonuniform. Combining the 4 materials in this study which are irregular shaped, and other materials investigated in Lei $[11,13,14]$, the following observations can be made: (1) Nonuniform spatial defect distributions are more often found in natural materials such as sugar, wood, coal, rocks and other mineral materials than in modern technological materials; Modern technological materials such as steels and classical concrete materials have been developed with a special focus on eliminating all nonuniform types for desired performance. (2) Porous structures are more susceptible to nonuniform defect distribution, such as wood, aluminum foam and some ceramics; (3) Materials easier to take inter-granular fracture than transgranular fracture, such as sugar, inter-metallics. It is interesting to note that wood present $\beta<1$ in tension along wood fiber direction but $\beta>1$ for tension normal to fiber direction. This means different types of defects play a role in each case. Right now, we are still in the early phase of accumulating "observations" on different materials [15-17]. In general, while it adds the complexity of problem solving, we do believe a nonuniform spatial distribution of defects in materials is a more realistic situation while a uniform spatial distribution is an idealized scenario; although an idealized distribution simplifies our effort to solve the problem, care should be taken that at some point we may hit the wall and have to take a major change of the uniform distribution based models. So, at this early phase, we would suggest to use the best fitting approach to determine $\beta$ value. As for the range of diameter or size, we believe for samples with larger diameter than those being considered here, the model should be still valid; however, for samples with much smaller size, fracture may involve some mechanism at a different size scale, the validity of the proposed approach has to be examined. As shown in Fig. 1, chronologically, the Weibull statistical strength theory was first adopted for size scaling of strength. Since Weibull statistical strength theory is a subordinate member of the uniform spatial flaw distribution based weakest link statistics, the work [12] applied the uniform spatial flaw distribution based weakest link statistics to study the size effect on particle breakage strength. For real materials, it is a more practical assumption that the spatial distribution of flaws can be uniform or non-uniform. The recent work [11] expanded the weakest link statistics to the more generic scenario in which the spatial flaw distribution in a material may be either uniform or non-uniform. This work collectively presents some examples to validate the applicability of power law spatial flaw distribution based weakest link model in particle breakage strength statistics. The contribution of this work lies in that it expands our capability to evaluate size scaling of particle breakage strength.

It is noted that for the 4 case studies presented in this work, the size dependence and the random variation of the breakage strength of a single particle are collectively represented by the following expression, which can be called as the generalized 2-parameter Weibull statistics:

$P\left(V, \sigma_{b}\right)=1-\exp \left[-\beta\left(\frac{V}{V_{0}}\right)^{\beta}\left(\frac{\sigma_{b}}{\sigma_{0}}\right)^{m}\right]$

However, at this point, we would not claim that Eq. (29) is applicable for all kinds of materials. The major purpose of this work is to verify and validate if the expected "master curve" for the $\frac{1}{\beta V^{\beta}} \cdot \ln \left[\frac{1}{(1-P)}\right]$ $\sim \sigma_{b}$ correlation exists or not. We expect that with rigorous statistical analysis, it is possible that either a generalized three-parameter Weibull distribution or other type of distribution functions may better fit the data for a specific material. The main limitation of the proposed procedure is for different materials, how to determine the fracture distribution of an element volume $\mathrm{V}_{0}, \mathrm{p}\left(\mathrm{V}_{0}\right)$, which affects the final expression of the cumulative probability $\mathrm{P}(\mathrm{V})$. In this study, if $\mathrm{g}(\mathrm{S})$, which is the PDF of fracture strength $\mathrm{S}$ of elemental volume $\mathrm{V}_{0}$, takes the Weibull PDF, an evident analytical solution of the cumulative probability $\mathrm{P}(\mathrm{V})$ can be obtained. However, one would argue that it is not necessary for all different materials the Weibull type PDF for $g(S)$ always works.

\section{Conclusions}

(1) The weakest link statistics based on the assumption of a power-law spatial distribution of flaws is validated for the size effect on 
breakage strength of rock aggregates, basalt, sugar and potash grains in diametrical compression. For each material, all the strength data of different sized particles fall onto the "master curve" for the $\frac{1}{\beta V^{\beta}} \cdot \ln \left[\frac{1}{(1-P)}\right] \sim \sigma_{b}$ correlation.

(2) For the four materials evaluated in this study, the value of $\beta$ ranges from 0.165 to 0.6 for the spatial flaw distribution function $(V)=\left(V / V_{0}\right)^{\beta}$.

\section{Acknowledgement}

This work was funded by the National Natural Science Foundation of China (No.11872364) and Chinese Academy of Science (CAS) Pioneer Hundred Talents Program.

\section{References}

[1] S. Aman, J. Tomas, H. Kalman, Breakage probability of irregularly shaped particles, Chem. Eng. Sci. 65 (2010) 1503-1512.

[2] Y. Rozenblat, D. Portnikov, A. Levy, H. Kalman, S. Aman, J. Tomas, Strength distribution of particles under compression, Powd. Technol. 208 (2011) 215-224.

[3] C. Ovalle, E. Frossard, C. Dano, W. Hu, S. Maiolino, P.-V. Hicher, The effect of size on the strength of coarse rock aggregates and large rock fill samples through experimental data, Acta Mech. 225 (2014) 2199-2216.

[4] L. Ribas, G.C. Cordeiro, R.D.T. Filho, L.M. Tavares, Measuring the strength of irregularly-shaped fine particles in a microcompression tester, Minerals Eng. 65 (2014) 149-155.

[5] S. Zhang, F. Baitalow, P. Nikrityuk, H. Gutte, B. Meyer, The effect of particle size on the strength parameters of German brown coal and its chars, Fuel 125 (2014)
200-205

[6] T. Afshar, M.M. Disfani, A. Arulrajah, G.A. Narsilio, S. Eman, Impact of particle shape on breakage of recycled construction and demolition aggregates, Powd. Technol. 308 (2017) 1-12.

[7] P.P. Cavalcanti, L.M. Tavares, Statistical analysis of fracture characteristics of industrial iron ore pellets, Powd. Technol. 325 (2018) 659-668.

[8] W. Weibull, A statistical theory of the strength of materials, Ingeniorsvetenskapakademiens, Handlinger 151 (1939) 1-45.

[9] W.-S. Lei, Evaluation of the basic formulations for the cumulative probability of brittle fracture with two different spatial distributions of microcracks, Fatigue Fract. Eng. Mater. Struct. 39 (2016) 611-623.

[10] W.-S. Lei, A framework for statistical modeling of plastic yielding initiated cleavage fracture of structural steels, Philos. Mag. 96 (2016) 3586-3631.

[11] W.-S. Lei, A generalized weakest-link model for size effect on strength of quasibrittle materials, J. Mater. Sci. 53 (2018) 1227-1245.

[12] Z. Yu, W.-S. Lei, J. Zhai, A synchronized statistical characterization of size dependence and random variation of breakage strength of individual brittle particles, Powder Technol. 317 (2017) 329-338.

[13] W. Lei, Statistical size scaling of ceramic strength, J. Am. Ceram. Soc. 102 (2019) 90-97.

[14] W. Lei, Fracture probability of a randomly oriented microcrack under multi-axial loading for the normal tensile stress criterion, Theor. Appl. Fract. Mech. 85 (2016) $164-172$

[15] G. Qian, W.-S. Lei, M. Niffenegger, V.F. Gonzalez, On the temperature independence of statistical model parameters for cleavage fracture in ferritic steels, Philos. Mag. 98 (2018) 959-1004.

[16] G. Qian, W.-S. Lei, L. Peng, Z. Yu, M. Niffengger, Statistical assessment of notch toughness against cleavage fracture of ferritic steels, Fatigue Fract. Eng. Mater. Struct. 41 (2018) 1120-1131.

[17] G. Qian, J. Zhai, Z. Yu, W. Lei, W. Wu, Non-proportional size scaling of strength of concrete in uniaxial and biaxial loading conditions, Fatigue Fract. Eng. Mater. Struct. 41 (2018) 1733-1745. 